\title{
EVALUACIÓN DE EXTRACTOS VEGETALES PARA EL CONTROL DE Mycosphaerella fijiensis EN PLÁTANO EN TIERRALTA - CÓRDOBA
}

\author{
EVALUATION OF PLANT EXTRACTS FOR CONTROLING \\ Mycosphaerella fijiensis IN PLANTAIN IN TIERRALTA - CORDOBA
}

\author{
Orlando J. Marín1, Marelis J. Mass, José L. Barrera¹ y Juana Robles² \\ Recibido para evaluación: Junio 18 de 2007 - Aceptado para publicación: Abril 15 de 2008
}

\begin{abstract}
RESUMEN
La investigación se realizó en Tierralta - Córdoba, durante los semestres B de 2005 y A de 2006. El objetivo del trabajo fue evaluar los efectos biofungicidas de varios extractos vegetales para el control de Mycosphaerella fijiensis en plátano (Musa AAB). Se establecieron dos experimentos, bajo condiciones de campo (época seca y época lluviosa) en un diseño completamente al azar. En el primer semestre se evaluaron 12 tratamientos (siete extractos vegetales, un caldo microbiológico, tres químicos y un testigo sin protección) con 5 repeticiones, realizándose aplicaciones y evaluaciones quincenales. En el segundo semestre se evaluaron los mejores tratamientos del primer experimento (seis extractos vegetales, el caldo microbiológico, un químico y un testigo sin protección) con aspersiones y evaluaciones semanales. La unidad experimental constó de 10 plantas de siete meses de edad (Inicio de beyoteo), a las cuales se les midió el número de hojas funcionales, hoja más joven enferma (YLI), hoja más joven con síntoma (YLS) e índice de infección (I.I.). En el primer experimento la enfermedad mostró sensibilidad a los extractos vegetales de Neem, Limoncillo, Salvia y Limoncillo + Neem superando al testigo en más del 7\%. En el segundo experimento los extractos vegetales presentaron un comportamiento similar al testigo químico, con índices de infección entre el 12 y $16 \%$, superando al testigo absoluto en $8 \%$. Se concluye, que a pesar de la efectividad de algunos productos químicos, el interés por conservar el ambiente y la creciente demanda de alimentos más sanos reconoce el control biológico con extractos vegetales como una estrategia de manejo fitosanitario.
\end{abstract}

Palabras claves: Mycosphaerella fijiensis, síntomas, severidad, biofungicidas.

1Universidad de Córdoba, Departamento de Ingeniería Agronómica y Desarrollo Rural, Carrera 6 No 76 - 103, MonteríaCórdoba. Telefax (4) 7860255 E-mail: jbarrera11@sinu.unicordoba.edu.co

${ }^{2}$ Universidad de Córdoba, Departamento de Matemáticas y Estadística. 
TEMAS AGRARIOS - Vol. 13:(1) Enero - Junio 2008 (25 - 31)

\begin{abstract}
This research was done in Tierralta - Cordoba, during semesters B 2005 and a 2006. The objective was to evaluate the biofungicidas effects of several vegetal extracts on controll ing of Mycosphaerella fijiensis in banana (Musa $A A B$ ). In order to reach it two experiments settled down, under conditions of field (Dry time and rainy time) in a design completely at random. First (2005-B) 12 treatments (seven vegetal extracts, a microbiological broth (boil), three chemistries and a witness without protection) with 5 repetitions were evaluated, being made biweekly applications and evaluations. In the second experiment (2006-A) the best treatments of the first experiment were evaluated (six vegetal extracts, biol, a chemistry (propiconazole) and a witness without protección). With weekly aspersions and evaluations. The experimental unit was composed by six plants of seven months of age (begin of beyoteo), to which the number of functional leaves was moderate to them, younger leaf becomes ill (YLI), younger leaf with symptom (YLS) and index of infection (I. I.). In the first experiment the disease showed sensitivity the vegetal extracts of Neem, Limoncillo, Salvia and Limoncillo+Neem surpassing to the witness in more of $7 \%$. In the second experiment the vegetal extracts presented/displayed a behavior similar to propiconazole, with indices of infection between 12 and $16 \%$, surpassing the witness in $8 \%$. It can conclude, that in spite of the atmosphere and the increasing demand of healthy foods recognizes the biological control (Vegetal Extracts) as a strategy of fitosanitario handling.
\end{abstract}

Key words: Mycosphaerella fijiensis, symptom, severity, biofungicides

\section{INTRODUCCIÓN}

En Colombia, el área sembrada en plátano es de 447.000 ha con una producción de 2.8 millones ton año-1 que representan el $10 \%$ de la producción mundial. De estos 100.000 ton año-1 se exportan y el resto es destinado al mercado nacional (Minagricultura, 2.000). El cultivo del plátano genera aproximadamente 142.516 empleos permanentes al año, lo que ocuparía un promedio de 36.000 familias en las labores del cultivo en todo el país (Rodríguez y Rodríguez, 1998). En Córdoba, hay establecidas 23.000 ha de plátano, que generan 10.000 empleos directos, con una producción de 7 ton ha-1 año-1 (Secretaría de Agricultura de Córdoba, 2002). El municipio de Tierralta tiene cultivadas 9.000 ha generando alrededor de 4.500 empleos directos.

Los productores de plátano del municipio de Tierralta presentan muchas desventajas en el desarrollo de dicha actividad, dado que esta es cada vez más competitiva y difícil de manejar por las crecientes exigencias de calidad, altos niveles de tecnificación y de protección ambiental, contrapuestos a una lucha contra plagas y enfermedades, cada día más difícil de manejar especialmente en el caso de la sigatoka negra. Todo esto ocasiona mayores niveles de inversión, difíciles de cumplir por los pequeños y medianos productores, trayendo como consecuencia la poca o nula comercialización a nivel internacional, ya que la fruta no reúne los estándares mínimos de calidad (EMC) exigidos por los exportadores (AUGURA, 2002). Pese a esta problemática, no se han realizado trabajos que busquen alternativas de producción de plátano de una manera más limpia y sostenible; es así, como la agricultura orgánica ofrece herramientas tales como la utilización ó implementación de extractos vegetales, para ser utilizados como biofunguicidas para el control de 
enfermedades en el cultivo del plátano. Con base en esto, se ha desarrollado el presente trabajo cuyo objetivo fue evaluar 12 tratamientos para el control de Sigatoka negra (Mycosphaerella fijiensis) en plátano (Musa $A A B)$ en el Municipio de Tierralta - Córdoba, con el fin de contribuir al mejoramiento ambiental de la zona, reduciendo el número de aplicaciones de fungicidas químicos utilizados actualmente para el control de dicha enfermedad.

\section{MATERIALES Y MÉTODOS}

El estudio se realizó durante el segundo semestre del 2005 y el primer semestre del 2006, en el municipio de Tierralta-Córdoba. Se utilizaron 12 tratamientos, con cinco repeticiones para la primera fase, mientras que para la segunda, se evaluaron los mejores nueve tratamientos de la fase anterior, con cinco repeticiones cada uno. Los tratamientos correspondieron a las aplicaciones de diferentes extractos vegetales utilizados como biofungicidas y controles absolutos y químicos (Tabla 1).

Para la elaboración de los extractos se colectaron en campo muestras del material vegetal a utilizar como hojas de balsamina (Momordica charantia), limoncillo (Swinglea glutinosa), salvia (Salvia officinalis), papaya (Carica papaya), neem (Azadirachta indica) y pringamosa (Jatropha sp.), las cuales se picaron y maceraron hasta obtener una masa; luego fueron depositadas en baldes plásticos con tapa, a cada balde se le agrego agua en proporción 2.5:1 (agua:muestra procesada, vol:peso), sometiéndola posteriormente a fermentación en un lugar limpio y seco por 78 horas, luego se procedió a colar y envasar el extracto para su posterior aplicación en campo. Como adherente natural en la aplicación de los extractos, se utilizó el aceite de higuerilla (Ricinus communis) en una concentración de 10 $\mathrm{cm}^{3} \mathrm{~L}^{-1}$ de mezcla, con el fin de evitar el fácil lavado de los productos naturales, ya que el tiempo del ensayo correspondió al período de Iluvias en la zona de estudio.

Tabla 1. Tratamientos evaluados sobre plantas de plátano (Musa AAB) en el semestre B de 2005 y A de 2006.

\begin{tabular}{|c|c|c|c|}
\hline \multicolumn{2}{|c|}{ Semestre B 2005} & \multicolumn{2}{|c|}{ Semestre A 2006} \\
\hline Tratamientos & Composición & Tratamientos & Composición \\
\hline T1 & Extracto de limoncillo $(2 \mathrm{~L})$ & $\mathrm{T} 1$ & Extracto de limoncillo (2 L) \\
\hline $\mathrm{T} 2$ & Extracto de papaya (2 L) & $\mathrm{T} 2$ & Extracto de papaya (2 L) \\
\hline T3 & Extracto de pringamoza $(2 \mathrm{~L})$ & T3 & Testigo \\
\hline $\mathrm{T} 4$ & Extracto de balsamina (2 L) & T4 & Extracto de balsamina (2 L) \\
\hline T5 & Extracto de limoncillo + & T5 & Extracto de limoncillo + \\
\hline & Extracto de Neem $(1 \mathrm{~L}+1 \mathrm{~L})$ & & Extracto de neem $(1 \mathrm{~L}+1 \mathrm{~L})$ \\
\hline T6 & Extracto de neem (2 L) & T6 & Extracto de neem (2 L) \\
\hline $\mathrm{T7}$ & Extracto de salvia (2 L) & $\mathrm{T7}$ & Extracto de salvia (2 L) \\
\hline T8 & Propiconazole $\left(1 \mathrm{~cm}^{3} \mathrm{~L}^{-1}\right)$ & T8 & Propiconazole $\left(1 \mathrm{~cm}^{3} \mathrm{~L}^{-1}\right)$ \\
\hline T9 & Jabón natural $(1$ cm³ L-1) & T9 & Biol + Bocachi $(1 \mathrm{~L}+1.5 \mathrm{Kg})$ \\
\hline $\mathrm{T} 10$ & Caldo bórdeles (1 $\left.\mathrm{g} \mathrm{L}^{-1}\right)$ & & \\
\hline $\mathrm{T} 11$ & Testigo & & \\
\hline $\mathrm{T} 12$ & Biol + Bocachi $(1.5 \mathrm{~L}+1.5 \mathrm{Kg})$ & & \\
\hline
\end{tabular}


Los tratamientos fueron distribuidos en el campo en un diseño completamente al azar, la unidad experimental estuvo constituida por seis plantas de siete meses de edad (inicio de diferenciación floral) y expuestas totalmente al sol. Tanto la aplicación de los tratamientos como la evaluación de los mismos se realizaron cada 15 días para la primera fase y cada 8 días para la segunda, durante 2.5 meses que es el tiempo que transcurre de la diferenciación floral a la emisión de bacota. Las variables evaluadas fueron número de hojas funcionales, la hoja más joven enferma (YLI), la hoja más joven con síntoma (YLS) e índice de infección (I. I.), para lo cual se utilizó el método de Stover modificado por Gauhl (1994). Los datos obtenidos fueron sometidos a análisis de varianza y a la prueba de comparación de medias de Tukey (1\%).

\section{RESULTADOS Y DISCUSIÓN}

\section{Número de hojas funcionales}

El análisis de varianza para el número de hojas funcionales durante la primera fase (semestre B - 2005), permitió detectar diferencias significativas $(P r<0.05)$, y la prueba de separación de medias Tukey señaló que el tratamiento químico (T8), fue superior al resto de los tratamientos (9 Hojas). Este resultado se debe muy probablemente a las bondades del producto por su efecto sistémico (Translaminar) (SYNGENTA, 2005), lo cual le permite brindar una mayor protección que los extractos vegetales (no sistémicos). Igualmente, se observó que el tratamiento seis (Neem) y el 12 (Biol) presentaron un promedio estadístico similar al químico (Tabla 2), permitiendo conservar hasta 8 hojas funcionales, que son suficientes para obtener racimos de buen peso y calidad (Barrera, 2004). Resultados muy similares se presentaron durante las evaluaciones de la segunda fase (semestre A 2006), donde el tratamiento químico continuó superando a los extractos con 9 hojas funcionales, esto indica que a pesar de que los extractos, al igual que el químico, tienen un efecto atenuante sobre la enfermedad, aumentar la frecuencia de aplicación ( 15 días a 7 días) no tienen un efecto significativo sobre esta variable (Tabla 2).

\section{Número de la hoja más joven enferma (YLS)}

El análisis de varianza reportó diferencias estadísticas altamente significativas entre tratamientos durante la primera fase del experimento. En la tabla 3, se observa que el tratamiento ocho (Químico) supera al resto de los tratamientos, al presentar menor severidad de la enfermedad. Se observó; sin embargo, que los tratamientos uno (Limoncillo), siete (Salvia), seis (Neen), y doce (Biol) presentaron una YLS número seis, la cual fue mayor a la del testigo sin aplicación (Hoja 5), al igual que presentaron medias estadísticas similares al testigo químico. En la segunda fase, se observa que los tratamientos ocho y seis resultaron en una YLS número ocho; mientras que los tratamientos uno, dos, cinco y nueve presentaron una YLS número siete, superando al testigo absoluto (YLS $=6$ ), lo cual indica que al igual que el producto químico los extractos vegetales tienen un efecto sobre la enfermedad atenuando su severidad y que este efecto se hace mas evidente al efectuar las aplicaciones con mayor frecuencia (semanal) (Tabla 2).

\section{Hoja más joven con síntomas (YLI)}

El análisis de varianza reportó diferencias estadísticas significativas entre los tratamientos para la variable hoja más joven con síntomas (YLI) durante la primera fase del experimento. Los datos consignados en la tabla 3 muestran que el tratamiento ocho supera al resto de los tratamientos al presentar una YLI número siete. Los tratamientos uno, siete y seis, presentaron una YLI número cinco, superando al testigo absoluto $(\mathrm{YLI}=4)$. En la segunda fase del experimento, los tratamientos seis y nueve 
Tabla 2. Influencia de diferentes tratamientos sobre las variables número de hojas funcionales (HF), hoja más joven enferma (YLS), hoja más joven con síntoma (YLI) y porcentaje de índice de infección en el control de Sigatoka Negra, semestre B-2005.

\begin{tabular}{|c|c|c|c|c|c|c|c|c|c|}
\hline \multicolumn{5}{|c|}{ Semestre B 2005} & \multicolumn{5}{|c|}{ Semestre A 2006} \\
\hline Tratamiento & HF & YLS & YLI & II & Tratamiento & HF & YLS & YLI & II \\
\hline 1 & $7.0 \mathrm{~b}$ & $6.0 \mathrm{~b}$ & $5.0 \mathrm{~b}$ & $28.0 \mathrm{bc}$ & 1 & $7.0 \mathrm{~b}$ & $7.0 \mathrm{ab}$ & $5.0 \mathrm{C}$ & $20.12 a$ \\
\hline 2 & $8.0 \mathrm{ab}$ & $5.5 \mathrm{bc}$ & $4.0 \mathrm{bc}$ & $33.6 \mathrm{ab}$ & 2 & $8.0 \mathrm{ab}$ & $7.0 \mathrm{ab}$ & $6.0 \mathrm{~b}$ & $17.86 \mathrm{a}$ \\
\hline 3 & $7.0 \mathrm{~b}$ & $5.0 \mathrm{bc}$ & $4.0 \mathrm{bc}$ & $36.5 \mathrm{ab}$ & 3 & $6.0 \mathrm{c}$ & $6.0 \mathrm{~b}$ & $5.0 \mathrm{C}$ & $22.53 a$ \\
\hline 4 & $6.5 \mathrm{~b}$ & $4.5 \mathrm{~cd}$ & $3,0 \mathrm{c}$ & $44.0 \mathrm{a}$ & 4 & $6.0 \mathrm{c}$ & $5.0 \mathrm{c}$ & $5.0 \mathrm{C}$ & $22.10 \mathrm{a}$ \\
\hline 5 & $7.0 \mathrm{~b}$ & $6.0 \mathrm{~b}$ & $4.5 \mathrm{bc}$ & $28.3 \mathrm{bc}$ & 5 & $7.0 \mathrm{~b}$ & $7.0 \mathrm{ab}$ & $6.0 \mathrm{~b}$ & 13.96a \\
\hline 6 & $7.0 \mathrm{~b}$ & $6.0 \mathrm{~b}$ & $5.0 \mathrm{~b}$ & $27.9 \mathrm{bc}$ & & $8.0 \mathrm{ab}$ & $8.0 \mathrm{a}$ & $7.0 \mathrm{ab}$ & 12.90a \\
\hline 7 & $7.0 \mathrm{~b}$ & $6.0 \mathrm{~b}$ & $5.0 \mathrm{~b}$ & $25.5 \mathrm{bc}$ & 6 & $6.0 \mathrm{c}$ & $6.0 \mathrm{~b}$ & $6.0 \mathrm{~b}$ & $15.86 a$ \\
\hline 8 & 9,0 a & $8.5 \mathrm{a}$ & $7.0 \mathrm{a}$ & 18. 2 c & 7 & 9,0 a & $8.0 \mathrm{a}$ & $8.0 \mathrm{a}$ & 15.73a \\
\hline 9 & $8.0 \mathrm{ab}$ & $6.0 \mathrm{~b}$ & $5.0 \mathrm{~b}$ & 29.4 bc & 8 & $7.0 \mathrm{~b}$ & $7.0 \mathrm{ab}$ & $7.0 \mathrm{ab}$ & 15.03a \\
\hline 10 & $7.0 \mathrm{~b}$ & $4.0 \mathrm{~d}$ & $4.0 \mathrm{bc}$ & $45.5 \mathrm{a}$ & 9 & & & & \\
\hline 11 & $7.0 \mathrm{~b}$ & $5.5 \mathrm{bc}$ & $4.0 \mathrm{bc}$ & $32.4 \mathrm{ab}$ & & & & & \\
\hline 12 & $8.0 \mathrm{ab}$ & $6.0 \mathrm{~b}$ & $4.5 \mathrm{bc}$ & $30.1 \mathrm{bc}$ & & & & & \\
\hline
\end{tabular}

Letras iguales no indican diferencias significativas según Tukey (1\%).

resultaron muy similares al tratamiento químico (ocho) obteniéndose una YLI número 7 y 8 , respectivamente (Tabla 2 ). Esto corrobora el hecho de que al cerrar lo ciclos de aplicación se hace más eficiente el control con los extractos, lo cual representa una hoja de diferencia traduciéndose en ganancia para la planta, dada la alta presencia de la enfermedad en la zona (Barrera, 2004).

\section{Índice de infección}

Con respecto esta variable durante la primera fase, el análisis de varianza reportó diferencias estadísticas significativas entre los tratamientos, observándose que el tratamiento químico (8) presentó el menor índice de infección del área foliar mientras que los extractos vegetales incidieron poco en la reducción de los valores de índice de infección (Tabla 2). Sin embargo, al finalizar el ensayo se encontraron índices de infección menores al 30\%, con los tratamientos uno (Limoncillo), siete (Salvia), cinco (Limoncillo + Neen) y seis (Neen), lo cual reitera el hecho de que el hongo Mycosphaerella fijiensis presenta susceptibilidad al uso de estos extractos vegetales. De forma inversa, el análisis de los resultados de la fase dos no mostró diferencias estadísticas significativas entre tratamientos, obteniéndose un índice de infección entre 13\% y $16 \%$ con tratamientos uno (Limoncillo), siete (Salvia), seis (Neen), ocho (Químico) y nueve (Biol),. Esto muestra claramente que con la aplicación de los extractos vegetales se logra un control similar al efecto del producto químico, cuando se reducen los ciclos de aplicación (Tabla 2). Resultados similares fueron obtenidos por Polanco y Rivero (2004), quienes encontraron que haciendo aplicaciones de Momordica charantia y Plenax sp a 20 ppm en plantas de plátano, la epidemia producida por Mycosphaerella fijiensis se retrasó de 12 a 15 días. Igualmente, Arciniegas y Riveros (2002) reportaron que extractos de Commelina difusa, Momordica charantia y Plenax sp in Vitro, mostraron una actividad antifúngica tanto en germinación de esporas como en desarrollo de colonias de Mycosphaerella fijiensis siendo en algunos casos mas efectivos que el propiconazole. 


\section{CONCLUSIONES}

- El Hongo Mycosphaerella fijiensis presenta susceptibilidad a las aplicaciones de extractos naturales de limoncillo (Swinglea glutinosa), salvia (Salvia officinalis), papaya (Carica papaya) y neem (Azadirachta indica), lo que permite que estos extractos puedan ser utilizados para el control de dicho patógeno en una producción limpia y sostenible del plátano.

- Las aplicaciones semanales de los extractos vegetales reducen la severidad del hongo Mycosphaerella fijiensis sobre las plantas de plátano, comparado con las aplicaciones quincenales, permitiendo conservar siete hojas funcionales al inicio de floración con índices de infección entre 13 y 16\%, los cuales no representan niveles de daño económico.
- Los tratamientos de los extractos naturales de Limoncillo (Swinglea g/utinosa), Salvia (Salvia officinalis), Papaya (Carica papaya), y Neem (Azadirachta indica) fueron estadísticamente similares al tratamiento químico con respecto al índice de infección, mientras que fueron estadísticamente mejores al compararse con tratamiento sin aplicaciones.

\section{AGRADECIMIENTOS}

Los autores expresan sus agradecimientos al Centro de Investigaciones de la Universidad de Córdoba (CIUC), por la financiación de este trabajo y a los profesores Rodrigo Campo Arana y Nuris Correa Genes, por su colaboración y dirección.

\section{BIBLIOGRAFÍA}

Arciniegas A. y Riveros A. 2002. Efecto de extractos vegetales sobre el desarrollo in Vitro de Mycosphaerella fijiensis, agente causal de la Sigatoka negra en Musáceas. Memorias XV reunión internacional ACORBAT, Cartagena, $617 p$.

AUGURA, Carta Informativa. Boletín 213. Octubre de 2002, Medellín Colombia, p1

Barrera, J. 2004. Contribución fisiológica de las hojas y el epicarpio del fruto en el llenado y calidad del racimo del plátano hartón (Musa AAB Simmonds) en el departamento de córdoba. Tesis M. Sc. Universidad Nacional de Colombia, Bogotá

CORBANA. 1994. Combate de la Sigatoka Negra. Boletín № 4. Departamento de Investigaciones, $21 \mathrm{pp}$.

Gauhl, F. 1994. Epidemiology and ecology of black sigatoka (Mycosphaerella fijiensis) on plantain and banana in Costa Rica, Central America. Tesis Ph. D., INIBAP, Montpellier

Minagricultura (Ministerio de Agricultura). 2000. Anuario Estadístico del Sector Agropecuario y Pesquero. Oficina de Información y Estadística, Bogota, D.C. 60 pp. 
Polanco D. y Riveros A. 2004. Evaluación en campo del potencial antifungico de extractos de plantas sobre Mycosphaerella fijiensis en Banano. Memorias XVI reunión internacional ACORBAT, Oaxaca, 282p.

Rodríguez, A. y Rodríguez, J. 1998. Aspectos socioeconómicos del cultivo del plátano en Colombia. CORPOICA, $35 \mathrm{pp}$.
Secretaria de Agricultura y Desarrollo Económico del Departamento de Córdoba. 2002. Anuario Estadístico. Gobernación de Córdoba, 23pp.

Stover, R. 1983. Effet du Cercospora noir sur les plantains en Amérique Centrale. Fruits 38:326-329.

SYNGENTA. 2005. Ficha Técnica Tilk. SYNGENTA, Bogota, p1 